\title{
Experimental Validation of a Hybrid Computational Model for Selective Stimulation Using Transverse Intrafascicular Multichannel Electrodes
}

\author{
Stanisa Raspopovic, Marco Capogrosso, Jordi Badia, Xavier Navarro, and Silvestro Micera, Senior Member, IEEE
}

\begin{abstract}
Recently a hybrid model based on the finite element method and on a compartmental biophysical representation of peripheral nerve fibers and intraneural electrodes was developed founded on experimental physiological and histological data. The model appeared to be robust when dealing with uncertainties in parameter selection. However, an experimental validation of the findings provided by the model is required to fully characterize the potential of this approach. The recruitment properties of selective nerve stimulation using transverse intrafascicular multichannel electrodes (TIME) were investigated in this work in experiments with rats and were compared to model predictions. Animal experiments were performed using the same stimulation protocol as in the computer simulations in order to rigorously validate the model predictions and understand its limitations. Two different selectivity indexes were used, and new indexes for measuring electrode performance are proposed. The model predictions are in decent agreement with experimental results both in terms of recruitment curves and selectivity values. Results show that these models can be used for extensive studies targeting electrode shape design, active sites shape, and multipolar stimulation paradigms. From a neurophysiological point of view, the topographic organization of the rat sciatic nerve, on which the model was based, has been confirmed.
\end{abstract}

Index Terms-Electrical neural stimulation, finite element method, intrafascicular time electrodes, model validation, rat axon model, rat sciatic nerve, selectivity.

\section{INTRODUCTION}

I MPLANTABLE peripheral neural interfaces have been used to develop neuroprosthetic systems that are able to improve the quality of life of disabled people [1], [2]. Different types of electrodes have been developed with the goal of achieving selective neural stimulation. They can be classified

Manuscript received September 05, 2011; revised December 31, 2011, January 25, 2012; accepted February 02, 2012. Date of publication April 03, 2012; date of current version May 18, 2012. This work was supported by the EU Grant CP-FP-INFSO 224012 (TIME project). S. Raspopovic and S. Micera equally contributed to this work.

S. Raspopovic and S. Micera are with the Translational Neural Engineering Lab, Center for Neuroprosthetics, Interfaculty Institute of Bioengineering (IBI), Ecole Polytechnique Federale de Lausanne (EPFL), CH-1015 Lausanne, Switzerland and also with the The BioRobotics Institute, Scuola Superiore Sant'Anna, 56127 Pisa, Italy.

M. Capogrosso is with The BioRobotics Institute, Scuola Superiore Sant'Anna, 56127 Pisa, Italy.

J. Badia and X. Navarro are with the Institute of Neurosciences, Department of Cell Biology, Physiology and Immunology, and Centro de Investigación Biomódica en Red sobre Enfermedades Neurodegenerativas (CIBERNED), Universitat Autónoma de Barcelona, E-08193 Bellaterra, Spain

Digital Object Identifier 10.1109/TNSRE.2012.2189021 according to their position with respect to the nerve structure: intraneural electrodes, including Utah slanted arrays [3], intraneural wires [4], [5] or intraneural silicon-shaft electrodes [6]; and epineural electrodes such as single channel [7], [8] and multiple-contact cuff [9]; and flat interface nerve electrodes [10], [11], among others.

Cuff electrodes have received wide attention in the past, since they are relatively easy to make and implant. Different types have been applied, showing to be safe for chronic use even in humans [12], [13]. Selective muscle contractions have been reported with spiral nerve cuff electrodes placed around the cat sciatic nerve [8], [9], [14], [15]. However, since they are placed around the nerve, epineural electrodes have limited selectivity for the stimulation of superficial fascicles in the nerve. By contrast, intraneural electrodes may allow more selective stimulation since they have more intimate contact with the different fascicles in both superficial and deep nerve locations. For example, promising results have been achieved in human studies with LIFE electrode implants [16], [17]. In both cases, an important research topic regards the optimization of the interface design aimed at improving performance, in terms of low stimulation threshold and high selectivity. For this reason, in the recent past, several groups have developed models addressing issues such as the identification of optimal interface design and positioning [18]-[21], optimal stimulation parameters and innovative waveforms for overcoming the inverse recruitment problem [22]-[26], and improvement of the efficiency of waveform shape [27].

We recently developed a hybrid model based on the finite element method (FEM) and on a compartmental biophysical representation of peripheral nerve fibers [28]. The model appeared to be quite robust with respect to uncertainties in parameter selection. However, it is crucial to validate the model by comparing the model predictions with real experimental results in order to fully characterize the potential of this approach. The goal of this paper is to address this exact issue. In particular, TIME electrodes [29] were used to stimulate the rat sciatic nerve, and electromyographic (EMG) signals were recorded from hindlimb muscles. Threshold currents, maximum muscle recruitment, muscle recruitment slopes, and muscle selectivity performance were compared with the results predicted from the model. The experimental validation is a very important step when developing effective and usable models since it can provide useful information for confirming or falsifying the underlying neurophysiological and physical assumptions. However, given its intrinsic complexity, only a few model validations have been carried out in this field in the past [11], [18], [19], [22], 


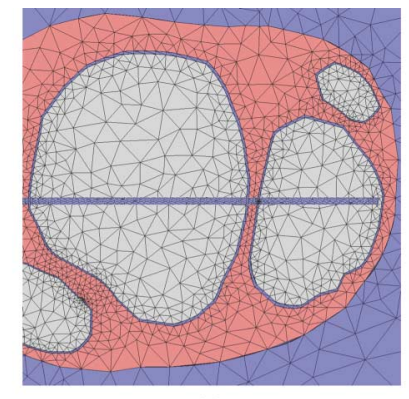

(a)

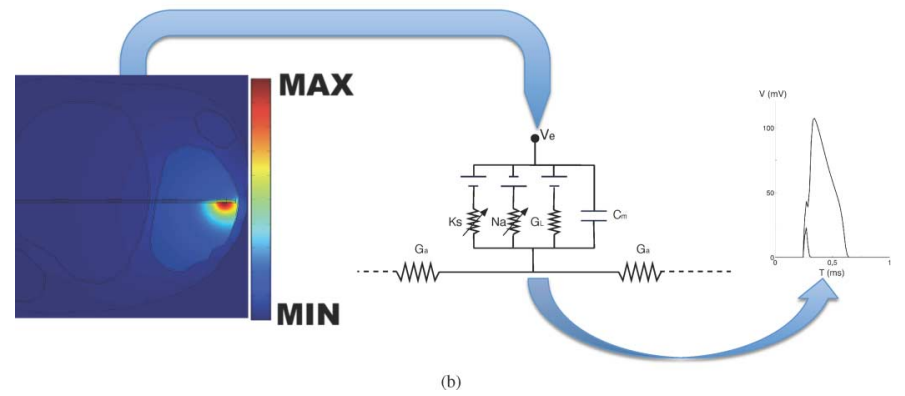

Fig. 1. Histological pictures and reconstruction of electrode positioning within the rat sciatic nerve for validation purpose. The nerve with the electrode inserted was harvested and fixed. Then $14-\mu \mathrm{m}$-thick transverse sections were made. Panels (a) and (b) show two serial sections in which the electrode can be seen traversing part of the nerve. Scale bar is $100 \mu \mathrm{m}$. In (c), the electrode trajectory was reconstructed and the regions in which the fibers innervating the three target muscles are located are depicted in different colors (MPN and LPN indicate median and lateral plantaris nerve, respectively, MG and LG indicate median and lateral gastrocnemius, TA indicate the Tibialis Anterioris): GM blue, PL yellow, and TA green. In red are schematically depicted the positions of the L1-L5 and R1-R5 active sites with respect to the muscle groups.

[24]. Generally, there is no evidence of strict experimental validation of model findings under the same conditions upon which the model is constructed. Particular attention was paid in this study to comparing the same conditions between the actual experimental tests and the model.

\section{MethodS}

\section{A. Hybrid Fem/Neuron Model}

A hybrid FEM/Neuron model of a rat sciatic nerve (Fig. 1) was developed and has been described in detail in a recent work [28]. In summary, it is an anatomically realistic (see Fig. 2) model, which also accounts for tissue conductivity anisotropy. It is based on a realistic volume conductor model of the rat sciatic nerve coupled with a fiber-cable model implemented in NEURON 7.1 [30]. The electrical stimulation problem was described by a Laplace formulation of the quasi-static Maxwell equations for the electric potential, sources were represented by currents injected through the electrode active sites

$$
\nabla \cdot \sigma \nabla V_{e}=0
$$

With Dirichlet boundary conditions set to zero at the outermost boundary of the model: $V_{e}(\delta \Omega)=0$, and a current density source $\mathbf{J}$ localized at the electrode active sites; $\sigma$ is the tissue specific electric conductivity as described in [28].

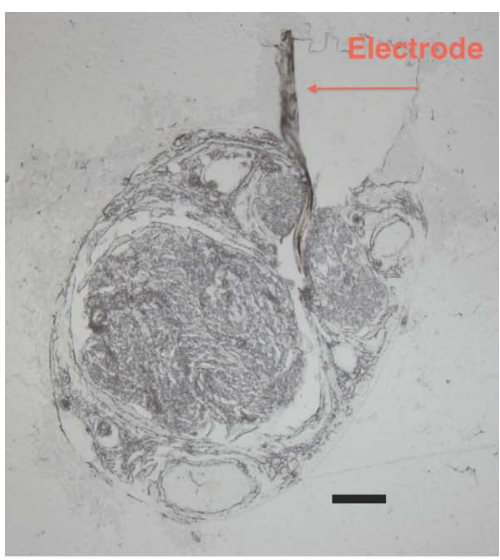

(a)

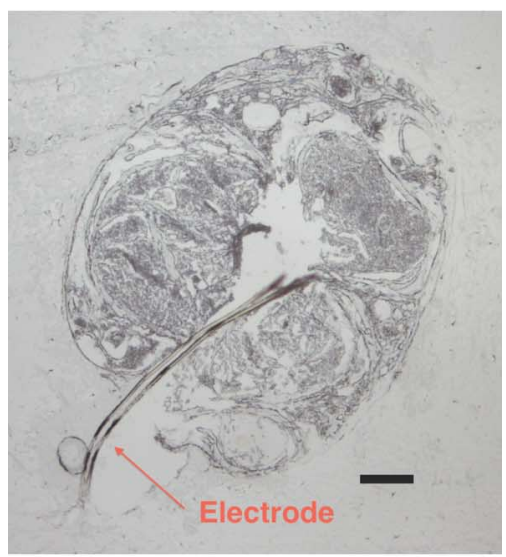

(b)

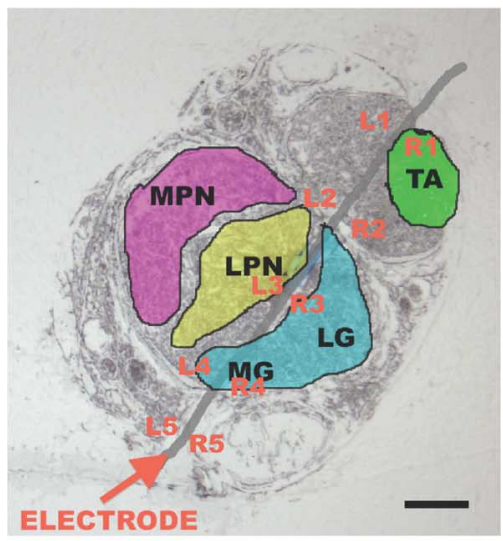

(c)

Fig. 2. The hybrid FEM/Neuron model. (a) Detail of the modeling of the rat sciatic nerve cross-section with high density mesh and the TIME electrode traversing the nerve (blue line), used to solve the electric field distribution. (b) Representation of the biophysics modeling: interpolation of the voltage solution onto the nodes of Ranvier and numerical solution of the Hodgkin-Huxley equations implemented for the rat axonal dynamics as in [28].

The finite element method was used to implement equations and geometries of the volume conductor model. The anatomical structures and the electrode were meshed using a non-uniform second order tetrahedral mesh, and all the finite element simulations were performed using COMSOL 3.5a (Comsol, Sweden). Numerical solutions for the electric potential $V_{e}$ were exported into MATLAB and interpolated onto the position of myelinated 
TABLE I

Electrical Properties of the Fiber Model [28]

\begin{tabular}{||l|l||}
\hline Property & Value \\
\hline \hline Nodal capacitance $\left(c_{m}\right)$ & $2 \mu \mathrm{F} / \mathrm{cm}^{2}$ \\
\hline Axoplasmic resistivity $\left(\rho_{a}\right)$ & $70 \Omega \mathrm{cm}$ \\
\hline Maximum $\mathrm{Na}^{+}$conductance $\left(g_{N a}\right)$ & $2.42 \mathrm{~S} / \mathrm{cm}^{2}$ \\
\hline Maximum $\mathrm{K}^{+}$conductance $\left(g_{K}\right)$ & $0.08 \mathrm{~S} / \mathrm{cm}^{2}$ \\
\hline Nodal leakage conductance $\left(g_{L}\right)$ & $0.34 \mathrm{~S} / \mathrm{cm}^{2}$ \\
\hline $\mathrm{Na}^{+}$Nernst rest potential $\left(E_{N a}\right)$ & $66 \mathrm{mV}$ \\
\hline $\mathrm{K}^{+}$Nernst rest potential $\left(E_{K}\right)$ & $-88 \mathrm{mV}$ \\
\hline Rest potential $\left(E_{\text {rest }}\right)$ & $-78 \mathrm{mV}$ \\
\hline
\end{tabular}

motor fibers and their nodes of Ranvier as described in [28]. A fiber-cable model made of 11 nodes of Ranvier and based on the experimental work of [31] was developed. The fiber model describes the Hodgkin-Huxley equations for the rat myelinated fiber membrane. Nodes of Ranvier were represented as active segments with sodium and potassium channels along with a leakage conductance. Dynamics of the membrane is fast and mainly sodium driven as experimentally described in [31]. Myelin was considered a perfect insulating sheath, therefore myelinated internodes were represented as purely resistive components. The modified Hodgkin-Huxley equations were implemented in NEURON 7.1 [30]. Electrical parameters are reported in Table I, state variable equations can be found in [28]. A fiber was considered recruited if an action potential travelled along its whole length [i.e., reached the last node of Ranvier, see Fig. 2(b)].

The anatomical structure was derived from histological sections taken from rat sciatic nerves used during the experiments. The electrode position was delineated from histological images obtained after dissection of the sciatic nerve in which the electrode was implanted (Fig. 1). The nerve fiber populations that innervate the gastrocnemius medialis (GM), the plantar interossei $(\mathrm{PL})$, and the tibialis anterior (TA) muscles, were overlaid taken into consideration their locations identified in a previous study [32] [see Fig. 1(c)].

In order to obtain a statistical distribution of possible electrode placements, the electrode was moved and rotated inside the nerve model obtaining nine different models [see Fig. 5(b)]. The electrode was moved by spanning the space of possible rotation angles and translational displacements from a standard central location. The constraint during the displacements was that all the active sites remained located inside the nerve, since this condition can be visually controlled by the surgeon. By changing the position of the electrode inside the nerve structure, the models are able to reproduce nine different stimulation experiments, with different relative distances and orientations between the active sites and the targeted fascicles. The population of models was used to statistically evaluate model performances versus experimental results.

\section{B. TIME Design}

The TIME electrode (Fig. 3) is made of a thin, strip-like polyimide structure that is folded during the implantation process in the same way as the tf-LIFE implantation [33]. This creates a double-sided electrode with 10 Platinum $(\mathrm{Pt})$ circular active sites (or poles), five for the left side (named L1 to 5) and five

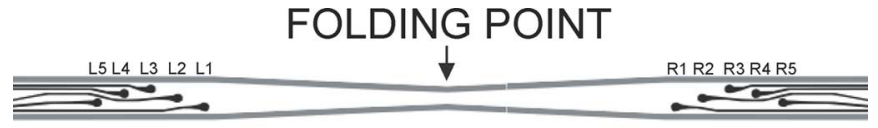

(a)

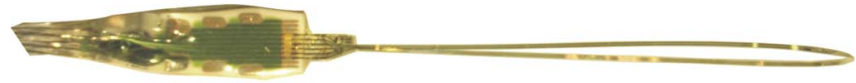

(b)

Fig. 3. (a) Design of TIME active sites used during the experiments and implemented in the computer model. (b) TIME electrode with the connector before the implantation.

for the right side (named R1 to 5, see for details [29]). Ten-polar TIME electrodes, with 10 zig-zag circular ring Pt electrodes, 60 $\mu \mathrm{m}$ in diameter [Fig. 3(a)] and intra-site distance of $230 \mu \mathrm{m}$ were used. The fabrication process and preliminary in vivo experiments have been previously described in detail [29].

\section{TIME Implantation and Stimulation Procedure}

Experimental data for this study was obtained from implants performed in five Sprague-Dawley rats (indicated as Rat 1 to Rat5). Under general anesthesia with pentobarbital $(40 \mathrm{mg} / \mathrm{kg}$ i.p.), the sciatic nerve was exposed at mid-thigh with the aid of microsurgery tools. The TIME was inserted transversally across the sciatic nerve as described in previous reports [29], [34] and was monitored under the dissection microscope to ensure that the electrode active sites were located inside the nerve tissue. After positioning the electrode, the thin-film loop was fixed to the epineurium of the nerve via a droplet of fibrin. The experimental procedures complied with the recommendations of the European Union and the NIH Guide for Care and Use of Laboratory Animals. They were also approved by the Ethical Committee of the Universitat Autonoma de Barcelona, where the animal tests were performed. The stimulation protocol was executed by a STIM'3D stimulator [35] that delivered programmed rectangular current pulses of $10 \mu \mathrm{s}$ duration and intensity ranging from 20 to $300 \mu \mathrm{A}$, at $0.5 \mathrm{~Hz}$, through each one of the ten different active sites of the TIME with respect to a small needle electrode placed on the nerve. The compound muscle action potentials (CMAPs) were recorded using needle electrodes placed in each target muscle, amplified (P511AC, Grass, West Warwick, RI), band-pass filtered (5 Hz to $2 \mathrm{kHz}$ ), and digitized with a PowerLab recording system (PowerLab16SP, ADInstruments, Bella Vista, Australia) at $20 \mathrm{kHz}$. The amplitude of each CMAP was measured from baseline to negative peak, and normalized to the maximal CMAP amplitude obtained for each muscle during the experiment [34]. The electromyographic (EMG) signals collected were fed into Matlab (The Mathworks, Inc., Natick, MA) for subsequent data analysis. EMG recordings were taken from the TA, GM, and PL muscles, which are innervated by different fascicles or subfascicles of the sciatic nerve [29]. The motor fibers innervating the TA muscle belong to the peroneal nerve branch, while fibers innervating GM and PL muscles belong to different subfascicles in the tibial nerve branch (Fig. 4). Thanks to this setup we aimed to assess stimulation selectivity at fascicular and sub-fascicular levels. 

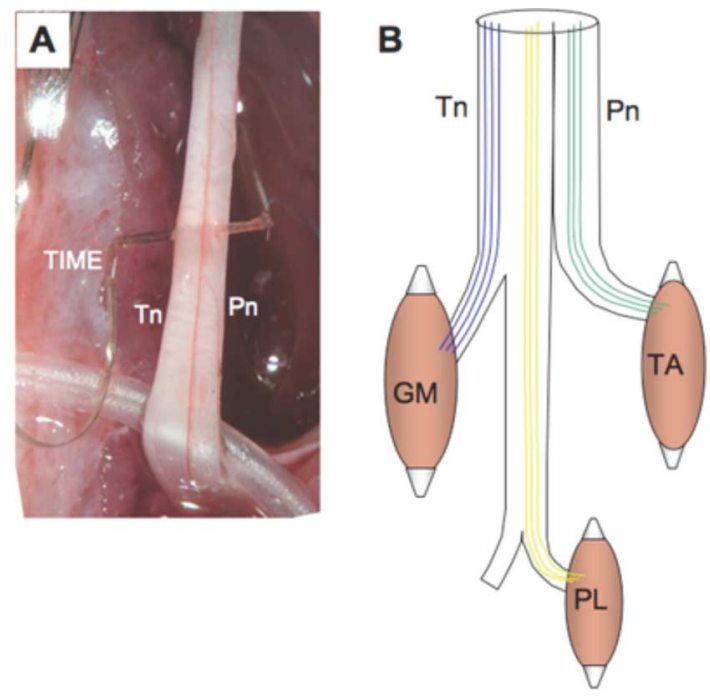

Fig. 4. (A) Photograph of a TIME implanted transversally across the tibial (Tn) and the peroneal (Pn) branches of the rat sciatic nerve. (B) Schematics of the experimental setup used to assess stimulation selectivity. The activation of TA, GM, and PL muscles was achieved when the stimulation excited the corresponding nerve fibers that run in separate bundles of the peroneal (TA) and tibial (GM and PL) nerve branches.

\section{Selectivity Indexes}

Selectivity is the ability of an electrode to elicit the activation of a targeted muscle without activating other undesired muscle groups. To assess this important feature of the electrodes, the following selectivity index was previously defined [28]:

$$
\operatorname{Sel}_{i}=\mu_{i}-\frac{1}{m-1} \sum_{j=1, j \neq i}^{m} \mu_{j}
$$

where $\mu_{i}$ is the percentage of recruitment of the $i$ th muscle group and $m$ is the total number of muscles targeted in the experiment. The recruitment percentage $\mu_{i}$ is defined as the number of activated fibers with respect to the total number of fibers innervating the $i$ th muscle in the case of a computer model. Instead, for experimental data, it is defined as the normalized value of the EMG signal (the CMAP in this case). In the experimental case, the $\mathrm{Sel}$ index is equivalent to the index defined in [36] except for the fact that thresholds are not used for percentage activation. It is comprised between -1 and 1 corresponding respectively to the situations of completely unselective and highly selective stimulation of targeted muscle. This index is compared experimentally to the commonly used SI [9]

$$
\mathrm{SI}_{i}=\frac{\mu_{i}}{\sum_{j=1}^{m} \mu_{j}} .
$$

The Sel and the SI indexes provide information about the ability of an electrode to stimulate a specific targeted muscle, therefore they can be defined as muscular selectivity indexes. Comparing two different electrodes by the use of one of these indexes alone would require a comparison of the performances for each targeted muscle. To have a comprehensive view of an electrode performance we defined three different "global" indexes $S_{T}, S_{e}$, and $S_{p}$. These indexes neither depend on the number of targeted muscles nor on the particular muscular selectivity index used to compute them. Their value is a score to be assigned to an electrode and is suitable for different electrode comparisons. For clarity the indexes are shown as computed using the Sel index for mucular selectivity, but any other index could be analogously used.

The $S_{T}$ index is defined as the average of maximum values of $\mathrm{Sel}_{i}$ for each muscle group reached during simulation

$$
S_{T}=\frac{1}{m} \sum_{i=1}^{m} \max \left(\operatorname{Sel}_{i}\right) .
$$

As an average, this index can provide important information about a mean selectivity value. However, it does not penalize cases in which selectivity for a single muscle is very low while others are very high. In order to overcome this problem, we defined the $S_{e}$ index which represent the normalized Euclidean distance of the computed selectivity with respect to the ideal case of $\mathrm{Sel}_{i}=1$ for each muscle

$$
S_{e}=1-\sqrt{\frac{1}{m} \sum_{i=1}^{m}\left(1-\max \left(\operatorname{Sel}_{i}\right)\right)^{2}} .
$$

In the "space" of muscular activation (with the number of dimensions equal to the number of considered muscles) the ideal electrode should be located near the vector with projection 1 on each muscle "axis" which would mean perfect selectivity for each muscle (e.g., $\mathrm{TA}=1, \mathrm{GM}=1, \mathrm{PL}=1$ in this case). The more the computed selectivity is distant from that vector, the poorer is the electrode performance. Therefore it is an intuitive (geometry-like) index to use. The index ranges between 0 and 1 , where 1 indicates perfect selectivity (e.g., $\max \left(\operatorname{Sel}_{i}\right)=1$ for each muscle) and 0 means no selectivity for any muscle. Finally, the $S_{p}$ index is defined as the m-root of the product of single maximal muscles selectivities

$$
S_{p}=\left(\prod_{i=1}^{m} \max \left(\operatorname{Sel}_{i}\right)\right)^{\frac{1}{m}} .
$$

This index is also comprised between 0 and 1 , where 0 means no selectivity and 1 means complete selective activation of all muscle subsets. In this index, 0 is also found if an electrode is not able to selectively stimulate one of the muscles; therefore this index is very penalizing. In this case, the targeted muscles could be diminished by one $(m-1)$.

The maximal selectivity values of $\mathrm{Sel}$ for each muscle were used to compare the computer model simulations with the experimental results. Values for each muscle were compared between the nine computer models, simulating the random insertion process of the electrode, and the five animal experiments. A Kruskal-Wallis test with a critical $p$-value of $p=0.01$ was performed to assess whether the experimental results were statistically distinguishable from the computer simulations. The Kruskal-Wallis test is an extension of the Wilcoxon rank-sum for data with more than two groups, and is suited for this type of analysis with a low number of observations.

\section{E. Model Validation: Fine Comparison}

The model was developed by using histological and functional data obtained from Rat1, which was used to perform the stimulation experiments and histological analysis. The ability 


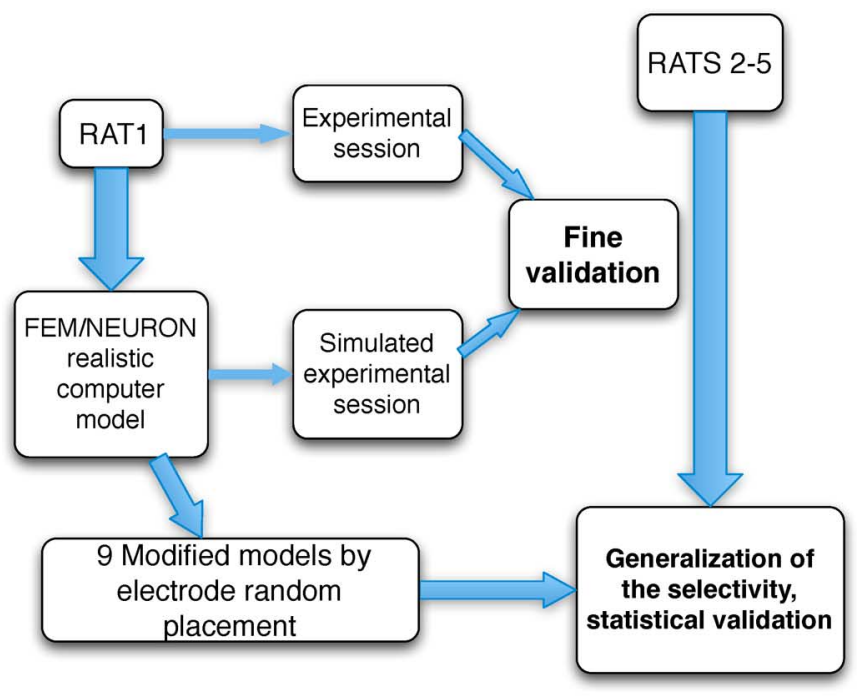

(a)

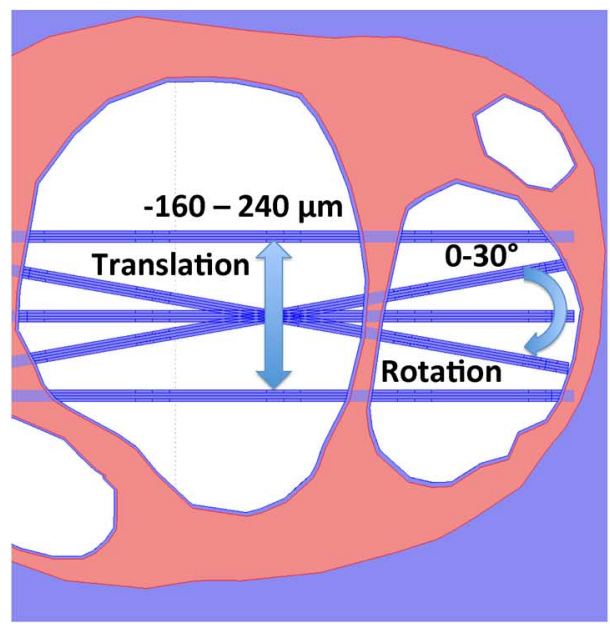

(b)

Fig. 5. Schematics of the validation process. (a) Model was developed using detailed anatomical information from RAT1, then a fine validation was obtained by reproducing the same experimental stimulation protocol. A generalization was achieved by reproducing the random insertion process of the electrode obtaining nine different models that were compared to the other experiments performed on different rats. (b) Representation of the simulated random insertion process.

of the model to reproduce real data and to understand to which extent it can be considered as a good approximation of reality can be evaluated by directly comparing the model-predicted and the experimental results on the same nerve. Recruitment curves were constructed in computer simulations and compared with the experimental results. Then, the selectivity properties of each of the 10 active sites of the TIME electrode were studied.

\section{F. Model Validation: Generalization}

Once the model was experimentally validated with data obtained for Rat1, its generalization properties with respect to new experiments (and different animals) were investigated. A scheme of the overall approach is given in Fig. 5. In this case we did not look for a high detail level such as the reproduction of the recruitment curves, as in the case of validation with data
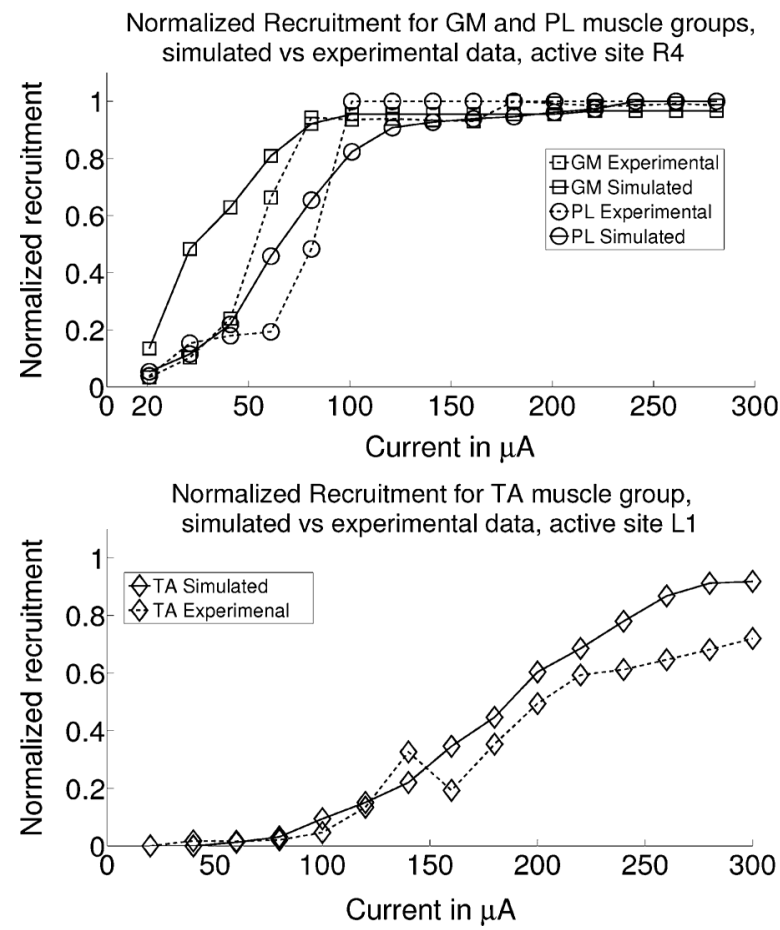

Fig. 6. Recruitment curves for the most selective active sites (experimental case). Dashed lines for experimental results, solid lines for simulations. Model appears to be in agreement with experimental findings.

from Rat1. Electrode insertion differences and anatomical and functional differences among animals make it impossible to have a one-to-one correspondence between active site locations within the model and the real nerve. Therefore, we tested the generalization ability of our model as the ability to reproduce the selectivity properties of the TIME electrode among a population of animals. For this reason, we performed simulations over different computer models built by varying the position and orientation of the electrode within the nerve according to the limits of movement found in [28] that reflect the maximal possible randomness of surgical insertion. According to the hypothesis of topographic functional repeatability, fiber groups inside the rat sciatic nerve are located in similar positions in every animal [32], [37]. For this reason, even if anatomical differences occur, results should be reproducible in terms of selectivity (which is the outcome for electrode efficacy). The $\mathrm{Sel}$ maximal selectivity values for each muscle were used to compare the computer model simulations with the experimental results as detailed in previous sections.

\section{RESULTS}

\section{A. Computer Model Validation: Simulated Versus Experimental Recruitment Curves}

Recruitment curves were constructed in simulations and compared with the experimental curves for the same Rat1. Then, the selectivity properties of each of the 10 active sites of TIME were studied. During the experiments the active sites L2 and L3 did not work properly (the traces leading to them where broken) and therefore resulted in no activation of any muscle. Recruitment curves for the most selective active sites are shown in Fig. 6. 


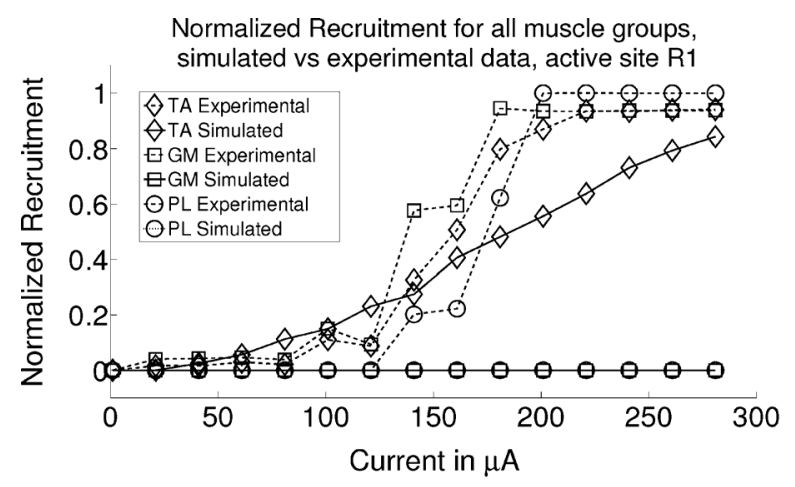

(a)

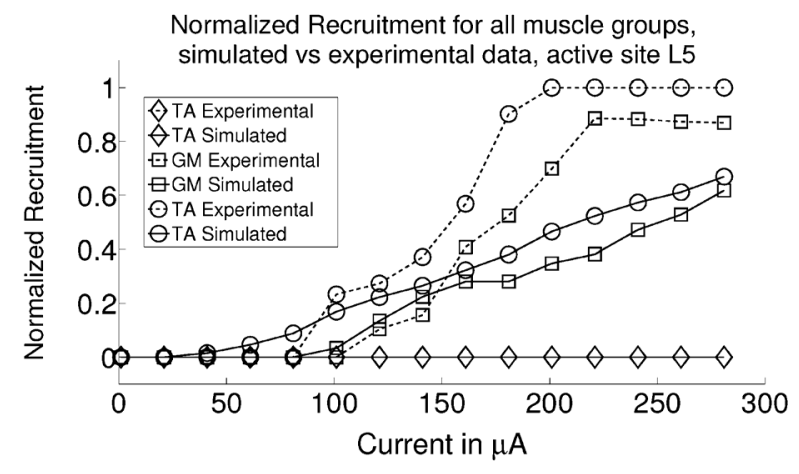

(b)

Fig. 7. Comparison of the non correctly predicted experimental conditions. The plots show recruitment curves for stimulation by active sites R1 and L5. In (a) the TA recruitment is reasonably well predicted, while PL and GM are missing. In (b) the model predicts onsets, but not the saturation. This kind of results are also very important for corrections of future models implementations.

The model shows good agreement with experimental data, both in terms of slope and activation onset. Since the data are taken from the most selective thus most useful active sites, the good agreement in terms of threshold and slope of the recruitment curve indicates the validity of the model hypothesis. The low onset currents, predicted from modeling are confirmed also in experimental data. However, it can be noticed that the model is not able to exactly reproduce the activation of the GM and PL muscle groups if stimulation is performed by "far" active sites (like R1) (see Fig. 7). Errors in slope and maximum activation are also present when stimulating from L5 active site (see Fig. 7). In this case, the active site is located near the selected muscle groups but actually lies outside the tibial fascicle perinerium. This can be the reason for the error in the recruitment properties.

The $\mathrm{Sel}$ selectivity index was computed for each of the 10 active sites. Maximum values of selectivity for each muscle and each active site are presented in Fig. 8, for experimental (dashed line) and model data (solid line).

At least one active site clearly exists for each muscle which elicits maximal selectivity and shows good concordance between the model and the experiment results (Fig. 8). Very high inter-fascicular selectivity is confirmed (if considering TA versus GM + PL), but also a good intrafasciular selectivity is found (PL versus GM) even using a high penalizing selectivity index.
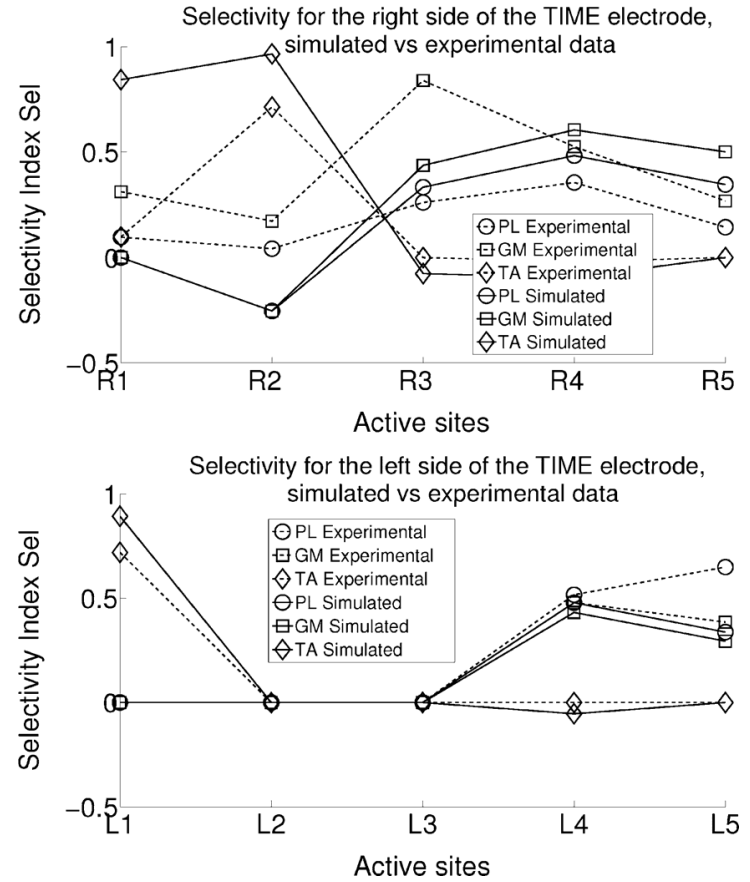

Fig. 8. Selectivity model predictions versus experimental findings. Simulated results are shown as dotted lines, while experimental results are solid lines. Active sites L2 and L3 did not function during the entire experiment.

Inverse recruitment is a well-known problem of FES devices. It consists in the activation of large motor nerve fibers before smaller ones, and is the opposite of physiological recruitment. Since the model is reasonably well in agreement with the experimental recruitment curves, we investigated the inverse recruitment properties of the model in order to estimate the amount of experimental inverse recruitment for the TIME electrode. The correlation coefficient $c$ between each fiber diameter and the corresponding current activation thresholds has been computed during the stimulation with the maximal selective active sites. A high negative value of $c$ would mean that larger diameter fibers are recruited at lower currents, while positive values would mean that larger fibers are recruited at larger currents e.g., after the smaller ones, which is the case of the natural recruitment. We performed a statistical analysis by changing randomly the positions of the fibers within the regions highlighted in Fig. 1. We obtained a value of $c=-0.26 \pm 0.1$ for the GM muscle, $c=-0.36 \pm 0.01$ for the PL, and $c=-0.89 \pm 0.06$ for the TA muscle. The negative values confirm the presence of an inverse relationship, which is lower for the stimulation of the PL and GM muscle than for the TA muscle. Fig. 9 reports normalized recruitment for each muscle and different diameter classes. Correlation coefficient values are reflected into this picture by the fact that in GM and PL (particularly at low currents) the amount of recruited large fibers (in yellow and light green) is not always higher than lower diameter. On the contrary, this does not happen for the TA which presents an approximately flat behavior for fibers larger than $7 \mu \mathrm{m}$. It means that in GM and PL some small fibers could be stimulated before the large ones, which is closer to the natural recruitment order. 


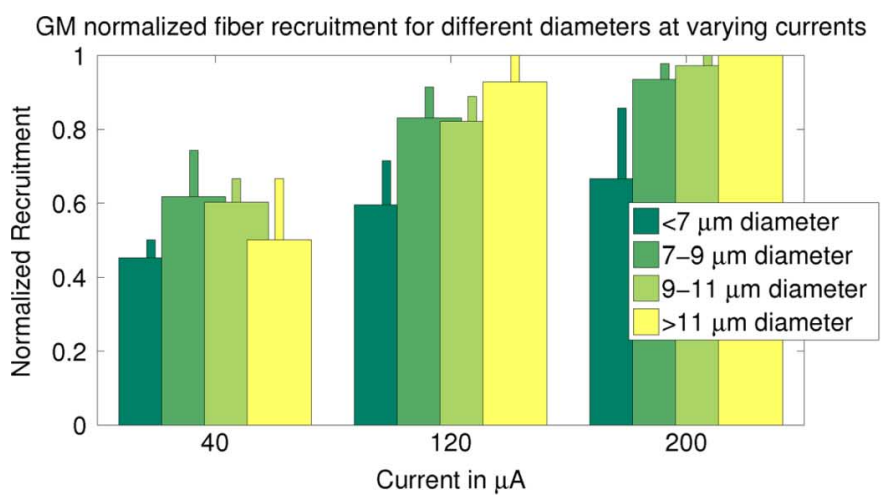

PL normalized fiber recruitment for different diameters at varying currents

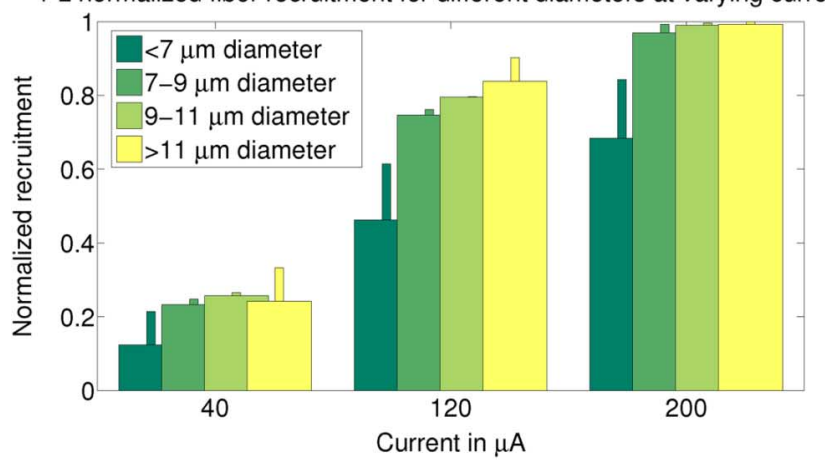

TA normalized fiber recruitment for different diameters at varying currents

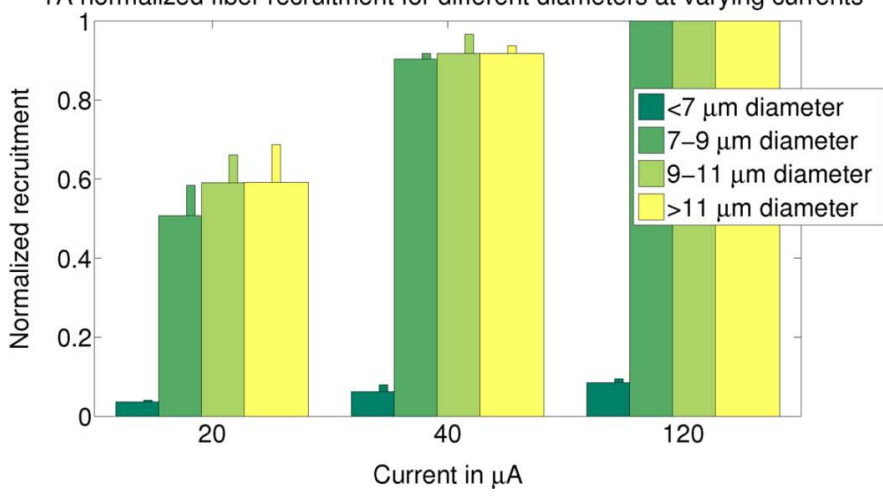

Fig. 9. Histograms representing the normalized recruitment for different fiber diameters at three current inputs for the stimulation with maximal selective active sites (e.g., L4 for PL, R4 for GM, L1 for TA), thinner bars represent maximum excursion from the average. The amount of inverse recruitment is smaller on the top plot (GM) and medium plot (PL), since it could be observed that for lower currents there is no strict monotonic order (meaning that first the larger fibers are elicited on lower currents), and even neither completely flat one (the larger are at least elicited together with smaller, which is yet inverse recruitment). This is particularly true for GM case. Instead for the TA (bottom plot) a clear inverse recruitment could be observed. The reasons for this are also discussed.

\section{B. Model Generalization}

The selectivity distributions achieved with our model, and compared with experiments with Rats 2-5 are shown in Fig. 10. The Kruskal-Wallis test showed no statistical difference between the model and the experiments $(p=0.16$ for PL, $p=$ 0.95 for GM, and $p=0.32$ for TA).

Both medians, as well as the spreads are similar between model (Sim) and experimental data (Exp) (see Fig. 10). The statistical analysis hence shows that the model results are in good agreement with the experimental data. This is a very important

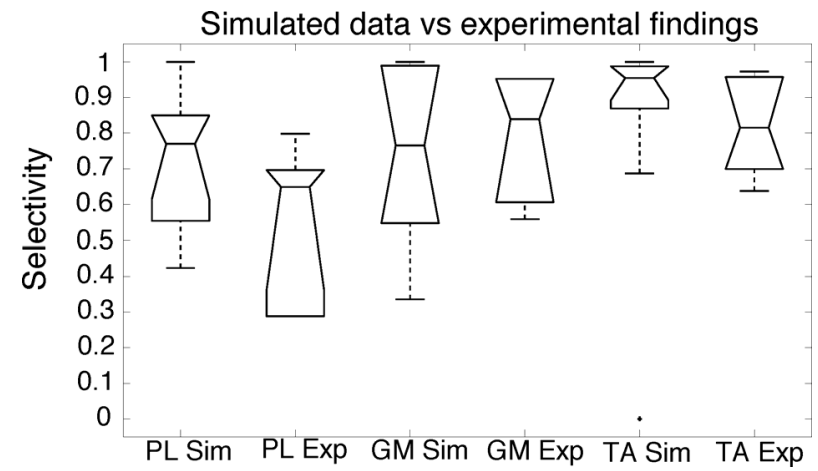

Fig. 10. Selectivity predictions from models versus experimental findings: statistical analysis. Boxes limits represent the 25 th and 75 th percentile of the data. The simulated selectivity values (Sim) are not statistically distinguishable from the experimental values (Exp), $p>0.01$. Crosses in the plot represent outliers. A data point is considered an outlier within the group if its value is further than three standard deviations from the average value. The horizontal line represents the median of the group.

outcome, since the model appears to be able to emulate the experimental selectivity, which is the most important feature for the electrode efficacy.

\section{Experimental Muscular and Global Selectivity}

Table II reports the computed maximum selectivity indexes for each muscle group and the global indexes $S_{T}, S_{e}$, and $S_{p}$, used to quantify the global electrode performance. Data are taken from the whole population of five rats. We computed these indexes using our definition of muscular selectivity, $\mathrm{Sel}$ (2), and the S.I. index proposed in the work [9], which has been often used in different versions in previous studies.

Because of its mathematical definition, the S.I. index can achieve a very high value also in cases in which the targeted muscle is very poorly contracted, and the untargeted muscles are not. In order to obtain a high value of $\mathrm{Sel}_{i}$ two conditions have to be satisfied. 1) The targeted muscle elicits enough contraction (normalized with respect to maximal value). 2) The untargeted muscles do not contract. The choice of the right index to be used depends on the particular application. If one is looking for little muscular forces then the S.I. index could fit, if one instead is interested in eliciting strong muscle force then the $\mathrm{Sel}_{i}$ index is better suited. From a global selectivity point of view the electrode achieves high results (see $S_{T}, S_{p}, S_{e}$ in Table II).

\section{DISCUSSION}

\section{A. Model Validation}

A hybrid FEM/Neuron model was developed according to anatomical and functional data gathered for a specific animal (Rat1). This allowed us to directly compare experimental data with simulation results (see Figs. 6 and 7). Electrode positioning inside the nerve was estimated from histological images of the implanted nerve. This made our computer model as close as possible to the real geometry. Direct comparison of recruitment curves for each muscle group was possible since there was a one-to-one correspondence between the real and simulated locations of the active sites within the nerve structure. The model 
TABLE II

Experimental Selectivity Properties of the Time Electrode, Data From a Population of Five Rats

\begin{tabular}{lcccccc}
\hline \hline Selectivity Indexes & PL & GM & TA & $S_{T}$ & $S_{e}$ & $S_{p}$ \\
\hline Sel $_{i}$ & $0.54 \pm 0.23$ & $0.78 \pm 0.18$ & $0.82 \pm 0.14$ & $0.71 \pm 0.03$ & $0.65 \pm 0.06$ & $0.67 \pm 0.04$ \\
S.I. & $0.72 \pm 0.21$ & $0.85 \pm 0.15$ & $0.94 \pm 0.06$ & $0.84 \pm 0.1$ & $0.79 \pm 0.13$ & $0.82 \pm 0.1$ \\
\hline
\end{tabular}

was able to reproduce the range of currents needed for excitation and a similar recruitment slope, although this parameter was underestimated in some cases. However, the model showed a limited prediction ability for the activation of GM and PL muscles when stimulating with active sites located within the peroneal nerve fascicle, and did not effectively reproduce the experimental values for these two muscles when active sites were located just outside the tibial nerve fascicle. This can be due to different factors, including an inaccurate model of the epineurium conductivity (this could be why the highest errors occurred when stimulating with active sites far from the innervation zones). It is also possible that, since the real electrode pierces the perineurium, liquids within the fascicles break out into the epineurium. This may have modified the isotropic conductivity properties that we used to model the tissue. Moreover, by piercing the perineurium, axons are no longer completely enclosed within a strong isolating structure, such as the perineurium [38], and this could have an impact (e.g., L5 active site stimulation). Detailed investigation (e.g., impedance measurements in vivo) of this specific parameter should be carried out in order to further improve the accuracy of our model. Nevertheless, the key factor for electrode efficacy, i.e., selectivity, was very well predicted with this model construction. We also studied the inverse recruitment properties of the TIME electrode within the model (testing the hypothesis that first large diameter and then small diameter fibers are recruited). We computed the correlation coefficient $c$ between the fiber diameter and the corresponding activation thresholds (as described in the previous sections). Correlation coefficients showed negative values, which indicate an inverse proportionality between the fiber diameter and the current threshold. However the values indicate a high-level of randomness in this inverse relationship. As expected for intraneural stimulation, the recruitment order is not strict. The inverse recruitment is more pronounced for TA muscle stimulation. This may depend on the fact that the functional nerve area is smaller than the others targeted, while for GM and PL the correlation coefficient is very low showing reduced inverse recruitment. If we consider that active sites are located inside the fascicles, the distance from the active sites becomes important just as the fiber diameter is important for the activation threshold, therefore, functional areas closer to active sites (for example, GM and PL) will show a more graded recruitment. Other studies on intraneural stimulation models reported similar results [39]. In a recent paper [40], the recruitment order of an innovative optical stimulation system was measured in the soleus and gastrocnemius muscle of mice, showing that optical stimulation is able to avoid the inverse recruitment relationship with respect to electrical stimulation. Our data confirm the existence of an inverse relationship in electrical stimulation experiments, however the model indicates that the TIME electrode is able to stimulate the GM muscle in rats eliciting a very low inverse recruitment $(c=-0.26 \pm 0.1)$, this is true in particular in the range of currents far from the saturation zone (40-120 $\mu \mathrm{A}$ see Fig. 9) which are the most likely to be used in real functional applications of the electrode.

\section{B. Model Generalization Ability}

The validation with Rat1 shows that the model is able to reproduce reasonably well experimental results when detailed histological and functional data are available. However, in most applications this is not the case. It is impossible to know, particularly in human applications, the specific location of the electrode contacts inside the nerve (since this would require dissection of that nerve). To a certain extent, therefore, the model should account for implantation blindness and variability of the anatomical topography. It should also be able to reproduce the most important feature of the electrode: selectivity. In [28] we showed that the implant model is robust for translations and rotations with respect to the central location. Therefore, in this paper, we computed the maximum selectivity values reached among nine models built by varying the position of the electrode inside the nerve. Then these values were compared with the experimental data regarding the same muscle groups. The statistical analysis showed that there are no significant differences between models and experiments for each muscle group, demonstrating that the models are able to generalize for selectivity. Moreover,, in [28] a sensitivity study over different anatomies is performed showing a maximum difference in the results of $10 \%$, which means that the position of the electrode introduces higher variability than the anatomy itself. Therefore once a general realistic anatomy model is implemented it can be used to infer hypotheses on the selectivity properties of an intraneural device without the need of customized models.

\section{TIME Electrode Selectivity Properties}

A novel method for characterizing selectivity has been defined in this manuscript and compared with the classical definition [9] commonly used in the field. Our Sel index is more penalizing than the classic S.I. index (see Table II). The bias of the S.I. towards high values is also reflected in the three global indexes ST, Se, and $\mathrm{Sp}$ which are higher than the same values computed using the Sel index. As expected the ST index is less penalizing, giving both for the Sel and S.I. higher values, while in the case of the TIME electrode, $\mathrm{Sp}$ and Se values are almost equivalent, this is because this type of electrode is able to reach high selectivity in all the targeted muscles. Nevertheless, in the case where one muscle would have had lower selectivity, then the difference in these two indexes would have been greater. It would be interesting to test this index for different electrodes on animal experiments or computer models. Consequently, it is useful when high selectivity is required for all the muscles involved and for different activation levels (e.g., the SI index tends to be already high for low activation levels, while $\mathrm{Sel}$ also accounts for a sufficient level of activation since it is normalized 
with respect to the maximal level of contraction). In particular, it is more penalizing to take into account the specific activation of muscle groups and to assign negative values to the activation of nontargeted muscle groups. We also defined three global indexes of selectivity, which can be considered as quality indexes for the electrode. As for the model [28] the experimental tests show that the TIME is able to elicit very high selectivity and to selectively stimulate every muscle with at least one active site, as previously demonstrated [34]. When data are compared between different studies, defining the selectivity index is essential for a correct interpretation of results. The specific index should always be described since results can vary significantly (see Table II). Finally, our results are an indirect confirmation of the topographic distribution of nerve fiber groups proposed in [32], which is the basic hypothesis of the present model. This is due to the fact that the TIME was able to stimulate different muscles with different active sites in every animal tested. The active sites are located in different geometrical positions inside the nerve, corresponding to the topographic innervation zones assumed.

\section{CONCLUSION}

Results achieved through animal experiments and computer models regarding recruitment and selectivity during the stimulation with TIME electrodes were compared. We showed that our model is able: 1) to reproduce reasonably well experimental recruitment curves when detailed topographic information is provided and 2) to predict TIME electrode selectivity for a population of animals, showing that the selectivity information inferred from the anatomically realistic model is generalizable. Finally, novel selectivity indexes both for individual muscles and for the whole electrode were used to show that the TIME electrode is able to reach very high selectivity values while eliciting a reduced inverse recruitment order of fibers as predicted by the model. Moreover, the neurophysiological topographic distribution of motor fascicles and subfascicles was indirectly confirmed.

\section{ACKNOWLEDGMENT}

The authors wish to thank Dr. T. Boretius and Prof. T. Stiegltiz (Department of Microelectronics, IMTEK, Freiburg, Germany) for fabricating the TIME electrodes. The authors acknowledge the technical help of J. Jaramillo in histological processing.

\section{REFERENCES}

[1] P. H. Peckham and J. S. Knutson, "Functional electrical stimulation for neuromuscular applications," Аnnu. Rev. Biomed. Eng., vol. 7, pp. $327-360,2005$.

[2] X. Navarro, T. B. Krueger, N. Lago, S. Micera, T. Stieglitz, and P. Dario, "A critical review of interfaces with the peripheral nervous system for the control of neuroprostheses and hybrid bionic systems," J. Peripher. Nerv. Syst., vol. 10, no. 3, pp. 229-258, Sep. 2005.

[3] A. Branner, R. B. Stein, and R. A. Normann, "Selective stimulation of cat sciatic nerve using an array of varying-length microelectrodes," $J$. Neurophysiol., vol. 85, no. 4, pp. 1585-1594, Apr. 2001.

[4] N. Nannini and K. Horch, "Muscle recruitment with intrafascicular electrodes," IEEE Trans. Biomed. Eng., vol. 38, no. 8, pp. 769-776, Aug. 1991.
[5] K. Yoshida and K. Horch, "Selective stimulation of peripheral nerve fibers using dual intrafascicular electrodes," IEEE Trans. Biomed. Eng., vol. 40, no. 5, pp. 492-494, May 1993.

[6] W. L. C. Rutten, H. J. van Wier, and J. H. M. Put, "Sensitivity and selectivity of intraneural stimulation using a silicon electrode array," IEEE Trans. Biomed. Eng., vol. 38, no. 2, pp. 192-198, Feb. 1991.

[7] G. G. Naples, J. T. Mortimer, A. Scheiner, and J. D. Sweeney, "A spiral nerve cuff electrode for peripheral nerve stimulation," IEEE Trans. Biomed. Eng., vol. 35, no. 11, pp. 905-916, Nov. 1988.

[8] J. D. Sweeney, D. A. Ksienski, and J. T. Mortimer, "A nerve cuff technique for selective excitation of peripheral nerve trunk regions," IEEE Trans. Biomed. Eng., vol. 37, no. 7, pp. 706-715, Jul. 1990.

[9] C. Veraart, W. M. Grill, and J. T. Mortimer, "Selective control of muscle activation with a multipolar nerve cuff electrode," IEEE Trans. Biomed. Eng., vol. 40, no. 7, pp. 640-653, Jul. 1993.

[10] D. J. Tyler and D. M. Durand, "Functionally selective peripheral nerve stimulation with a flat interface nerve electrode," IEEE Trans Neural Syst. Rehabil. Eng., vol. 10, no. 4, pp. 294-303, Dec. 2002.

[11] D. K. Leventhal and D. M. Durand, "Chronic measurement of the stimulation selectivity of the flat interface nerve electrode," IEEE Trans. Biomed. Eng., vol. 51, no. 9, pp. 1649-1658, Sep. 2004.

[12] K. H. Polasek, H. A. Hoyen, M. W. Keith, R. F. Kirsch, and D. J. Tyler, "Stimulation stability and selectivity of chronically implanted multicontact nerve cuff electrodes in the human upper extremity," IEEE Trans. Neural Syst. Rehabil. Eng., vol. 17, no. 5, pp. 428-437, 2009.

[13] L. E. Fisher, D. J. Tyler, J. S. Anderson, and R. J. Triolo, "Chronic stability and selectivity of four-contact spiral nerve-cuff electrodes in stimulating the human femoral nerve," J. Neural Eng., vol. 6, no. 4, p. 046010, Aug. 2009.

[14] W. M. Grill and J. T. Mortimer, "Quantification of recruitment properties of multiple contact cuff electrodes," IEEE Trans. Rehabil. Eng., vol. 4, no. 2, pp. 49-62, Jun. 1996.

[15] M. D. Tarler and J. T. Mortimer, "Selective and independent activation of four motor fascicles using a four contact nerve-cuff electrode," IEEE Trans. Neural Syst. Rehabil. Eng., vol. 12, no. 2, pp. 251-257, Jun. 2004.

[16] G. S. Dhillon, T. B. Krüger, J. S. Sandhu, and K. W. Horch, "Effects of short-term training on sensory and motor function in severed nerves of long-term human amputees," J. Neurophysiol., vol. 93, no. 5, pp. 2625-2633, May 2005.

[17] P. M. Rossini, S. Micera, A. Benvenuto, J. Carpaneto, G. Cavallo, L. Citi, C. Cipriani, L. Denaro, V. Denaro, G. D. Pino, F. Ferreri, E. Guglielmelli, K.-P. Hoffmann, S. Raspopovic, J. Rigosa, L. Rossini, M. Tombini, and P. Dario, "Double nerve intraneural interface implant on a human amputee for robotic hand control," Clin. Neurophysiol., vol. 121, no. 5, pp. 777-783, May 2010.

[18] T. A. Frieswijk, J. P. Smit, W. L. Rutten, and H. B. Boom, "Force-current relationships in intraneural stimulation: Role of extraneural medium and motor fibre clustering," Med. Biol. Eng. Comput., vol. 36, no. 4, pp. 422-430, July 1998.

[19] K. E. Deurloo, J. Holsheimer, and P. Bergveld, "Nerve stimulation with a multi-contact cuff electrode: Validation of model predictions," Arch. Physiol. Biochem., vol. 108, no. 4, pp. 349-359, Oct. 2000.

[20] Z. Lertmanorat and D. M. Durand, "Extracellular voltage profile for reversing the recruitment order of peripheral nerve stimulation: A simulation study," J. Neural Eng., vol. 1, no. 4, pp. 202-211, Dec. 2004.

[21] M. A. Schiefer, R. J. Triolo, and D. J. Tyler, "A model of selective activation of the femoral nerve with a flat interface nerve electrode for a lower extremity neuroprosthesis," IEEE Trans. Neural Syst. Rehabil. Eng., vol. 16, no. 2, pp. 195-204, Apr. 2008.

[22] Z.-P. Fang and J. T. Mortimer, "Selective activation of small motor axons by quasitrapezoidal current pulses," IEEE Trans. Biomed. Eng., vol. 38, no. 2, pp. 168-174, Feb. 1991.

[23] W. M. Grill and J. T. Mortimer, "Stimulus waveforms for selective neural stimulation," IEEE Eng. Med. Biol. Mag., vol. 14, no. 4, pp. 375-385, Jul./Aug. 1995.

[24] W. M. Grill and J. T. Mortimer, "Inversion of the current-distance relationship by transient depolarization," IEEE Trans. Biomed. Eng., vol. 44, no. 1, pp. 1-9, Jan. 1997.

[25] A. Vuckovic, N. J. M. Rijkhoff, and J. J. Struijk, "Different pulse shapes to obtain small fiber selective activation by anodal blocking-a simulation study," IEEE Trans. Biomed. Eng., vol. 51, no. 5, pp. 698-706, May 2004.

[26] K. Hennings, L. Arendt-Nielsen, S. S. Christensen, and O. K. Andersen, "Selective activation of small-diameter motor fibres using exponentially rising waveforms: A theoretical study," Med. Biol. Eng. Comput., vol. 43, no. 4, pp. 493-500, Jul. 2005. 
[27] A. Wongsarnpigoon, J. P. Woock, and W. M. Grill, "Efficiency analysis of waveform shape for electrical excitation of nerve fibers," IEEE Trans. Neural Syst. Rehabil. Eng., vol. 18, no. 3, pp. 319-328, Jun. 2010.

[28] S. Raspopovic, M. Capogrosso, and S. Micera, “A computational model for the stimulation of the rat sciatic nerve using a transverse intrafascicular multichannel electrode," IEEE Trans. Neural Syst. Rehabil. Eng., vol. 19, no. 4, pp. 333-344, Aug. 2011.

[29] T. Boretius, J. Badia, A. Pascual-Font, M. Schuettler, X. Navarro, K. Yoshida, and T. Stieglitz, "A transverse intrafascicular multichannel electrode (time) to interface with the peripheral nerve," Biosensors Bioelectron., vol. 26, no. 1, pp. 62-69, 2010.

[30] M. L. Hines and N. T. Carnevale, "The neuron simulation environment," Neural Comput., vol. 9, no. 6, pp. 1179-1209, Aug. 1997.

[31] J. R. Schwarz and G. Eikhof, "Na currents and action potentials in rat myelinated nerve fibres at 20 and 37 c," Pflugers Arch, vol. 409, no. 6, pp. 569-577, Aug. 1987.

[32] J. Badia, A. Pascual-Font, M. Vivó, E. Udina, and X. Navarro, “Topographical distribution of motor fascicles in the sciatic-tibial nerve of the rat," Muscle Nerve, vol. 42, no. 2, pp. 192-201, 2010.

[33] N. Lago, K. Yoshida, K. P. Koch, and X. Navarro, "Assessment of biocompatibility of chronically implanted polyimide and platinum intrafascicular electrodes," IEEE Trans. Biomed. Eng., vol. 54, no. 2, pp. 281-290, Feb. 2007.

[34] J. Badia, T. Boretius, D. Andreu, C. Azevedo-Coste, T. Stieglitz, and $\mathrm{X}$. Navarro, "Comparative analysis of transverse intrafascicular multichannel, longitudinal intrafascicular and multipolar cuff electrodes for the selective stimulation of nerve fascicles," J. Neural Eng., vol. 8, no. 3, 2011.

[35] D. Andreu, D. Guiraud, and G. Souquet, "A distributed architecture for activating the peripheral nervous system," J. Neural Eng., vol. 6, no. 2, p. 026001, Apr. 2009

[36] M. A. Schiefer, K. H. Polasek, R. J. Triolo, G. C. J. Pinault, and D. J. Tyler, "Selective stimulation of the human femoral nerve with a flat interface nerve electrode," J. Neural Eng., vol. 7, no. 2, Apr. 2010.

[37] D. Prodanov and H. K. P. Feirabend, "Morphometric analysis of the fiber populations of the rat sciatic nerve, its spinal roots, and its major branches," J. Comp. Neurol., vol. 503, no. 1, pp. 85-100, Jul. 2007.

[38] Y. Grinberg, M. A. Schiefer, D. J. Tyler, and K. J. Gustafson, "Fascicular perineurium thickness, size, and position affect model predictions of neural excitation," IEEE Trans. Neural Syst. Rehabil. Eng., vol. 16, no. 6, pp. 572-581, Dec. 2008.

[39] P. H. Veltink, J. A. Van Alste, and H. B. K. Boom, "Simulation of intrafascicular and extraneural nerve stimulation," IEEE Trans. Biomed. Eng., vol. 35, no. 1, pp. 69-75, 1988.

[40] M. Llewellyn, K. Thompson, K. Deisseroth, and S. Delp, "Orderly recruitment of motor units under optical control in vivo," Nature Med., vol. 16, no. 10, pp. 1161-1166, 2010.

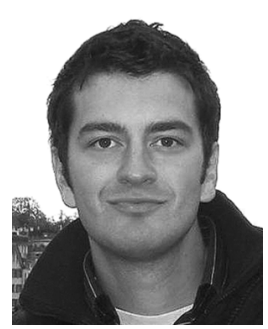

Stanisa Raspopovic received the B.S. and M.S. degrees (Hons.) from the Electrical and Biomedical Engineering faculty at University of Pisa, Pisa, Italy, in 2007, and Ph.D. degree in biomedical robotics at the Scuola Superiore Sant' Anna, Pisa, Italy, in 2011.

During 2007 and 2009, he was a Visiting Researcher at the Universitat Autonoma Barcelona, Spain, working on in vivo experimentation with peripheral nerve electrodes, for stimulation and decoding, and in 2010 at ETH Zurich, Zurich, Switzerland, working on the electrical epidural stimulation for the spinal cord injury. His research interests are in neural engineering: neural interfaces implants in animal and human models (both in peripheral nerve and in spinal cord), hybrid neural modeling, processing of the peripheral and central nervous system signals, for understanding the neural control of movement and prostheses control.

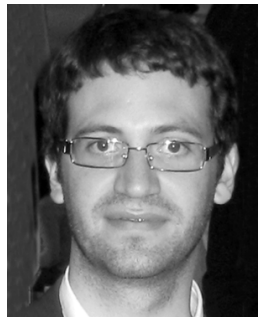

Marco Capogrosso received the B.S. degree in physics and the M.S. degrees in applied physics (cum laude) from the Department of Mathematical, Physical and Natural Sciences, the University of Pisa, Pisa, Italy. He is currently working toward the Ph.D. degree in biorobotics at the Institute of Biorobotics, Scuola Superiore Sant'Anna, Pisa, Italy.

His research interests are in computational models for neuroscience and neural engineering: neuroprostheses in animal and human models for the peripheral stimulation of the nervous system and spinal cord stimulation techniques.

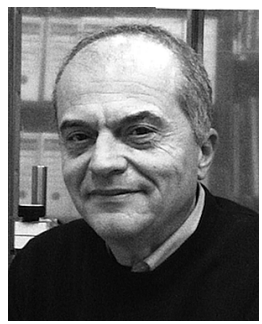

Jordi Badia received the degree in the physics, in 2000 , the M.S. degree in neurosciences, in 2007, and the Ph.D. degree in neuroscience, in 2011, from the Universitat Autònoma de Barcelona.

$\mathrm{He}$ is a Research Fellow in the Group of Neuroplasticity and Regeneration of the Institute of Neurosciences, Universitat Autònoma de Barcelona His research has been centered in the investigation of neurobiological effects and electrophysiological characterization of interfaces for the peripheral nervous system.

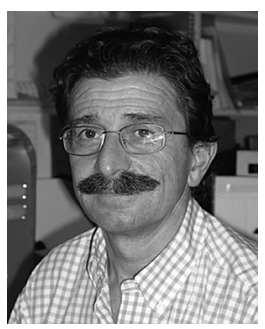

Xavier Navarro received the M.D. degree, in 1978, and the Ph.D. degree in medicine, in 1985, from the Universitat Autònoma de Barcelona. He completed speciality training in neurology at the University of Barcelona, and in neurophysiology at the University of Minnesota while holding a Fulbright fellowship (1986-1988).

He is presently Full Professor of Physiology a the Universitat Autònoma de Barcelona. From 1989 he is heading the research group on Neuroplasticity and Regeneration of the Institute of Neurosciences. He serves also as scientific advisor of the Institute Guttmann for Neurorehabilitation. His research interests are focused on axonal regeneration, functional restitution after neural injuries, neuroprostheses, and peripheral neuropathies.

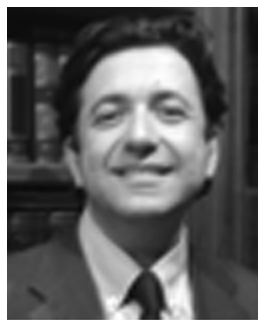

Silvestro Micera (SM'06) received the University degree (Laurea) in electrical engineering from the University of Pisa, Pisa, Italy, in 1996, and the Ph.D. degree in biomedical engineering from the Scuola Superiore Sant'Anna, Italy, in 2000.

During 1999, he was a Visiting Student at the Aalborg University. From 2000 to 2009 , he has been an Assistant Professor of BioRobotics at the Scuola Superiore Sant'Anna where he is now the Head of the Neural Engineering Group. In 2007 he was a Visiting Scientist at the Massachusetts Institute of Technology, Cambridge, with a Fulbright Scholarship. From 2008 he is the Head of the Neuroprosthesis Control Group and an Adjunct Assistant Professor at the Institute for Automation, Swiss Federal Institute of Technology, Zurich, CH. His research interests include the development of hybrid neuroprosthetic systems (interfacing the central and peripheral nervous systems with artificial systems) and of mechatronic and robotic systems for function and assessment restoration in disabled and elderly persons. He is author of several scientific papers and international patents. He served as Guest Editor of several biomedical engineering journals. He is a member of the Editorial Board of the Journal of Neuroengineering and Rehabilitation.

Dr. Micera was the recipient of the "Early Career Achievement Award" of the IEEE Engineering in Medicine and Biology Society in 2009. He is currently Associate Editor of IEEE TRANSACTIONS ON BIOMEDICAL ENGINEERING and of IEEE TRANSACTIONS ON NEURAL SySTEMS AND REHABILITATION ENGINEERING. He is Deputy Editor in Chief of the IEEE EMB Magazine. 